\title{
EVALUACIÓN DE LA DISTRIBUCIÓN DEL ESPACIO EN LA PLANTA INCUBADORA ESPAM-MFL MEDIANTE EL ALGORITMO CORELAP
}

\section{EVALUATION OF THE SPACE DISTRIBUTION IN THE ESPAM- MFL INCUBATOR PLANT USING THE CORELAP ALGORITHM}

María Isabel Delgado Moreira, María Virginia Moreira Macías, Diana Beatriz Vidal Zambrano, Yomaly Melissa Andrade Macías, Carlos Ricardo Delgado Villafuerte

Carrera de Ingeniería Ambiental, Escuela Superior Politécnica Agropecuaria de Manabí Manuel Félix López, Campus Politécnico El Limón, Km 2.7 Vía Calceta - El Limón

Email: mariai.delgado@espam.edu.ec

\section{Información del artículo}

Tipo de artículo:

Nota técnica

Recibido:

20/05/2020

Aceptado:

14/05/2021

Licencia:

CC BY-NC-SA 4.0

Revista:

ESPAMCIENCIA

12(1):69-74

DOI:

https://doi.org/10.51

260/revista_espamci

encia.v12i1.201

\section{Resumen}

La distribución del espacio causa un alto impacto en el rendimiento de cualquier industria o cadena de procesos. Por ello, el objetivo de esta investigación fue evaluar la distribución en planta de la incubadora de la Escuela Superior Politécnica Agropecuaria de Manabí Manuel Félix López mediante el algoritmo CORELAP. La metodología empleada, se basa en el método SPL (Systematic Layout Planning). Se identificaron los productos para determinar el orden que llevan los movimientos de los materiales a lo largo de diversas etapas del proceso. Se elaboró un plano con base en la actual distribución de la planta Incubadora mediante el software AutoCAD 2018. Como resultado, se detallan los nueve procesos que se realizan en la incubadora. Se determinó que la distribución en la planta incubadora es de procesos y mediante un diagrama relacional se constató que varias de las relaciones entre las operaciones no tienen importancia entre sí. La aplicación del algoritmo CORELAP demostró que, la actual distribución de la incubadora es adecuada. El enfoque presentado en este estudio es susceptible de usarse en la mejora de los niveles de producción de plantas incubadoras.

Palabras clave: Planta de incubación, CORELAP, proceso, diagrama, distribución en proceso

\begin{abstract}
The distribution of space causes a high impact on the performance of any industry or process chain. Therefore, the objective of this research was to evaluate the plant distribution of the incubator at Escuela Superior Politécnica Agropecuaria de Manabí Manuel Félix López using the CORELAP algorithm. The methodology used is based on the SPL method (Systematic Layout Planning). Products were identified to determine the order of material movements through various stages of the process. A plan was prepared based on the current layout of the incubator using AutoCAD 2018 software. As a result, nine processes carried out in the incubator were detailed. It was determined that the distribution in the hatchery is of processes and by means of a relational diagram it was found ouy that several of the relationships between the operations are not important to each other. The application of the CORELAP algorithm showed that the current incubator distribution is adequate. The approach presented in this study can be used to improve hatchery production levels.
\end{abstract}

Keywords: Hatchery, CORELAP, process, diagram, distribution in process 


\section{INTRODUCCIÓN}

Para Jung y Lee (2019) y Mejía et al. (2011) la distribución en planta es la organización física de los factores y elementos que participan en el proceso productivo de la empresa y la determinación de espacios y ubicación de sus distintas secciones. Por su parte, Ejeh et al. (2019); Yang y Chih (2007) destacan que la distribución en planta invariablemente tiene un impacto significativo en el rendimiento de un sistema de fabricación o industria de servicios y, en consecuencia, ha sido sujeto de investigación activa durante varias décadas.

El problema del proceso de distribución en la planta implica decisiones relacionadas con la asignación espacial de los elementos del equipo y las conexiones necesarias entre ellos (De Lira, et al., 2019; Patsiatzis y Papageorgiou, 2002). Por lo general, estas decisiones de distribución de la planta se ignoran o no reciben la atención adecuada durante el diseño o la modernización (Akhila y Deepthia, 2019; Ning et al., 2019).

Latifi et al. (2017); Guirardello y Swaney (2005) recalcan que la distribución cuidadosa de los equipos y materiales en una planta incubadora es importante desde un punto de vista económico, así como por razones de seguridad, operativas y de mantenimiento.

La incubadora de pollos Broiler (BB) de la ESPAM MFL, inició actividades en el año 2008 con 2350 pollos de raza Ross 308. Actualmente, la incubadora produce 2500 pollitos BB mensualmente y cuenta con cuatro trabajadores. En tal sentido, infiriendo que la distribución de las instalaciones del establecimiento en estudio es la más conveniente, se planteó como objetivo evaluar la distribución del espacio en la planta incubadora ESPAM MFL mediante el algoritmo CORELAP.

\section{MATERIALES Y MÉTODOS}

\section{Ubicación}

El estudio se realizó en el campus politécnico de la ESPAM MFL, localizada en el sitio El Limón, parroquia Calceta, cantón Bolívar-Manabí, en las coordenadas 0049'27,9''S; 80¹0'47.2'’O, a 15 msnm.

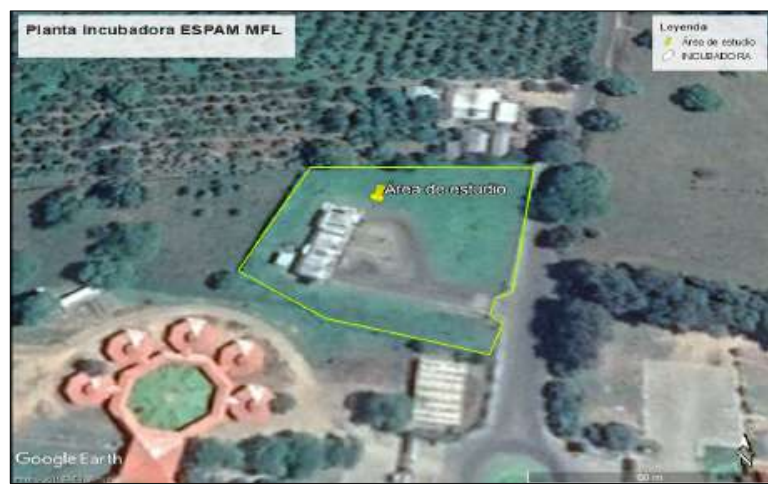

Figura 1. Ubicación de la Planta Incubadora de pollitos BB ESPAM MFL

Para el desarrollo de esta investigación se empleó el método analítico, atendiendo a la naturaleza descriptiva de la misma; empleándose técnicas como entrevistas y recolección de datos in situ. Además, se aplicó el método SLP (Systematic Layout Planning) desarrollado por Muther (1968).

\section{Procedimiento}

El desarrollo de esta investigación se realizó acorde a las etapas descritas a continuación:

Determinación del flujo de proceso y tiempos requeridos en cada una de las actividades que se realizan en la Incubadora ESPAM MFL

Se realizó un análisis del recorrido de los productos, que implica la determinación de la secuencia de los movimientos de los materiales a lo largo de diversas etapas del proceso, utilizando la simbología propuesta por Muther (1968) que se especifica a continuación:

Cuadro 1. Símbolos para realizar un diagrama de procesos

\begin{tabular}{cll}
\hline Símbolo & $\begin{array}{c}\text { Tipo de } \\
\text { acción }\end{array}$ & Resultado predominante \\
\hline$\square$ & Operación & Producir o realizar \\
$\square$ & Transporte & Desplazar \\
$\square$ & Control & Verificar \\
$\square$ & Espera & Interferir \\
$\square$ & Almacenaje & Conservar \\
& Combinada & $\begin{array}{l}\text { Indica varias actividades } \\
\text { simultáneas }\end{array}$ \\
\hline
\end{tabular}

Fuente: Muther (1968) 
Dado que este diagrama no es un factor que determina el emplazamiento del proceso de operaciones, simplemente indica sobre un gráfico la secuencia de operaciones, determinando cuáles son los departamentos que necesitan estar próximos (González, 2002). Se realizó un análisis de las relaciones entre actividades según lo propuesto por Muther (1968) mediante el cual se integró las actividades y procesos. Esto permitió evaluar la importancia de la proximidad entre las actividades, apoyándose sobre una codificación apropiada.

Así mismo, se identificaron las corrientes residuales de la incubadora.

\section{Elaboración de un plano de la distribución en planta de la incubadora ESPAM MFL}

Después de identificar cada uno de los procesos realizados en la incubadora de pollitos BB ESPAM MFL se realizó un análisis para las actividades de producción en general, en el que se incluyó los procesos y el tiempo (Ojagh et al., 2005). Después, se elaboró un plano de la actual distribución en planta utilizando el software AutoCAD 2018 (Autodesk, 2018).

Evaluación de la distribución en planta de la Incubadora ESPAM MFL

Para evaluar la actual distribución en planta de la incubadora de pollitos BB ESPAM MFL se empleó el algoritmo CORELAP que Mejía et al. (2011) describen como una técnica tradicional utilizada en la construcción de distribuciones cuyo acrónimo significa Computarized Relationship Layout Planning (CORELAP), el cual fue desarrollado en 1967, siendo uno de los pioneros en el campo de la distribución asistida por computador.

En esta metodología se ubican los departamentos de acuerdo con la calificación de cercanía total representada en trayectoria rectilínea, siendo el de mayor relación de cercanía situado en el centro de la disposición y como regla de desempate siempre se selecciona el departamento de área más grande (Mejía et al., 2011).

\section{RESULTADOS Y DISCUSIÓN}

Mediante recorridos guiados en la incubadora de pollitos BB ESPAM MFL se determinó que dicha instalación posee el ordenamiento mostrado a continuación:

Cuadro 2. Secuencia de actividades de la Incubadora de Pollos BB ESPAM MFL.

\begin{tabular}{clc}
\hline Número & Actividad u operación & Área $\mathbf{( m}^{\mathbf{2}} \mathbf{)}$ \\
\hline 1 & Recepción de huevos & 21,53 \\
\hline 2 & Clasificación de huevo fértil & 17,24 \\
\hline 3 & Sala de frío & 16,30 \\
\hline 4 & Incubadora & 20,11 \\
\hline 5 & Nacedora & 14,00 \\
\hline 6 & $\begin{array}{l}\text { Clasificación y vacunación de } \\
\text { pollitos }\end{array}$ & 19,50 \\
\hline 7 & Embalaje & 19,17 \\
\hline
\end{tabular}

Con la información obtenida se elaboró el diagrama de procesos (Figura 2).

Dicho diagrama, muestra que el tipo de distribución en planta de la incubadora es una distribución por procesos o como mencionan Okpala y Chukwumuanya (2016) conocida también como distribución funcional, este tipo de distribución es muy adecuada para aplicaciones donde los productos que se obtienen de las materias primas y el trabajo en curso implican grandes variaciones durante el procesamiento de las operaciones individuales. Muther (1968) recalca que esta distribución es muy útil en situaciones donde el proceso de producción está estructurado en lotes ya que los diferentes productos están organizados para moverse de un área a otra, en función de la sucesión de operaciones.

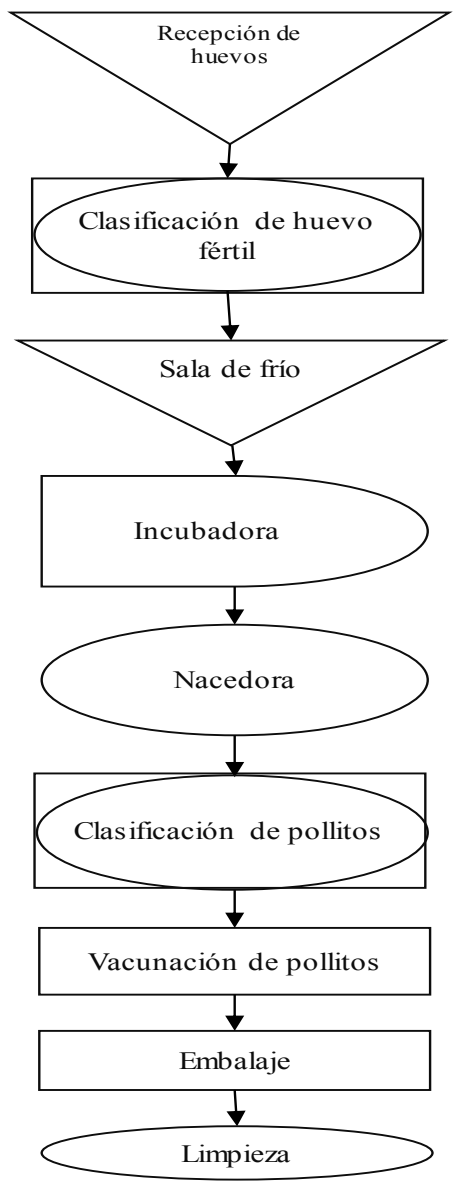

Figura 2. Diagrama de procesos de la Incubadora de pollitos BB ESPAM MFL

Además, se elaboró el diagrama relacional de la incubadora (Figura 3). El cual muestra que varias de las relaciones entre las operaciones de la incubadora no tienen importancia. Sin embargo, aquellas que son fundamentales se encuentran en prioridades adecuadas (absolutamente necesario o especialmente necesario). 


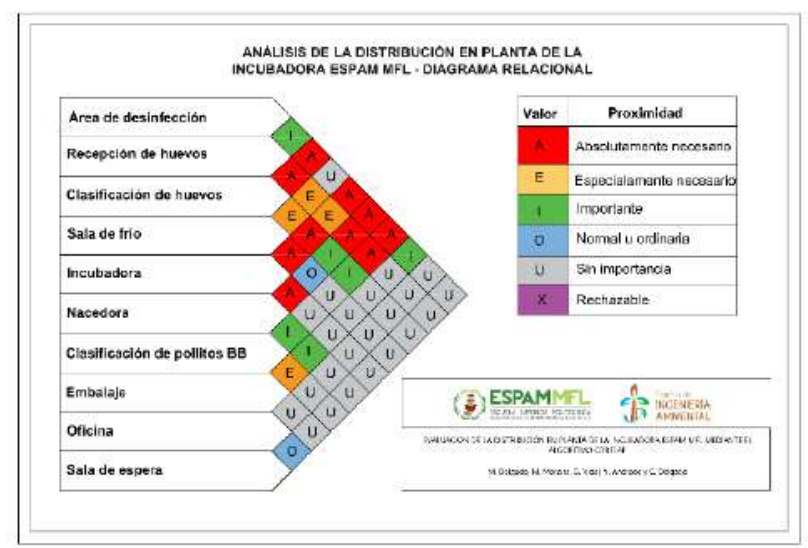

Figura 3. Diagrama relacional Incubadora de pollitos BBB ESPAM MFL

Tal como mencionan Ali et al. (2016) mediante el diagrama relacional establece la decisión de posicionamiento relativo entre las áreas funcionales; además, ofrece una visión general de la relación de cercanía junto con restricciones prácticas y actúa como prioridad para las alternativas de diseño.

Para realizar el plano de la distribución en planta de la Incubadora ESPAM MFL se recopilaron datos de la producción mensual de la incubadora. El tiempo de entrega de un lote de pollitos $\mathrm{BB}$ es de 39 días; a continuación, se muestran los tiempos en cada uno de los procesos (Cuadro 3):
Cuadro 3. Tiempos de las operaciones unitarias en la incubadora

\begin{tabular}{clc}
\hline Número & \multicolumn{1}{c}{ Actividad u operación } & $\begin{array}{l}\text { Tiempo } \\
\text { (días) }\end{array}$ \\
\hline 3 & Sala de clasificación de huevo & 12 \\
& fértil & 5 \\
4 & Sala de frío & 19 \\
5 & Incubadora & 2 \\
6 & Nacedora & 0,5 \\
7 & Sala de clasificación y vacunación & 0,5 \\
\hline 8 & de pollitos BB & Sala de embalaje \\
\hline
\end{tabular}

En la actualidad, la incubadora recepta 5040 huevos cada 15 días, teniendo una producción de pollitos final de aproximadamente 2500 pollitos mensuales, en dicha producción se generan las corrientes residuales descritas a continuación:

En la incubadora de pollos ESPAM MFL no existe gestión de desechos sólidos, éstos son recolectados por el servicio municipal. Según estimaciones de Delgado et al. (2013) los desechos biológicos son lo que se producen en mayor porcentaje $(93 \%)$ y se genera un $7 \%$ de desechos comunes (cartón, papel, cinta de embalaje, etcétera). También se generan desechos peligrosos (jeringas y envases de productos veterinarios vacíos) en un mínimo porcentaje (Figura 4).

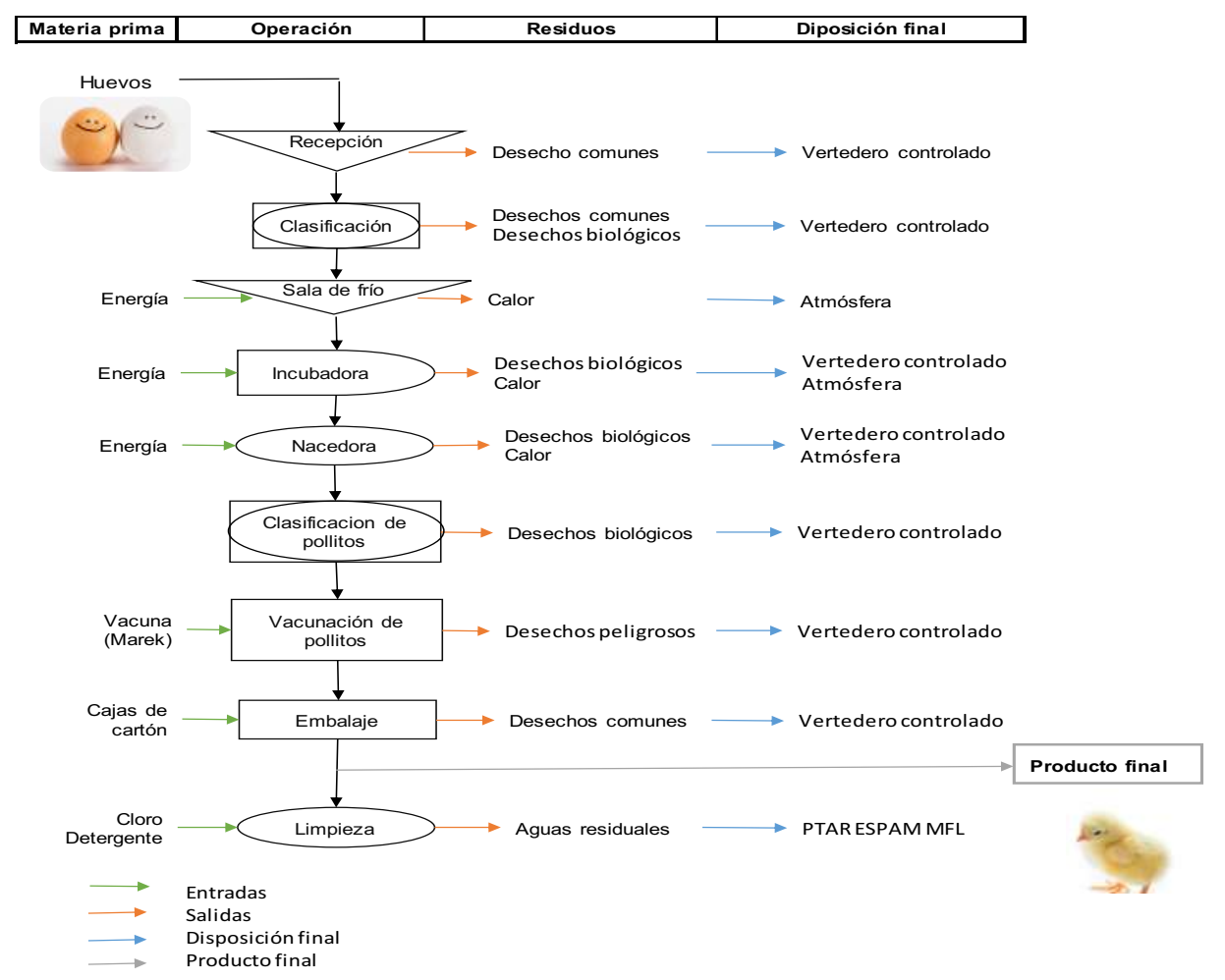

Figura 4. Corrientes residuales identificadas en la producción de la Incubadora ESPAM MFL 
Todos estos desechos se colocan en contenedores para que la recolección municipal realice la disposición final de los mismos.

En cuanto a las aguas residuales generadas, cabe recalar que la ESPAM MFL posee una planta de tratamiento
(PTAR) para disminuir la carga contaminante de todas sus actividades; por lo que, la planta incubadora de la ESPAM vierte los residuos líquidos en dicha planta.

Las corrientes residuales se incluyeron en el plano realizado (Figura 5).

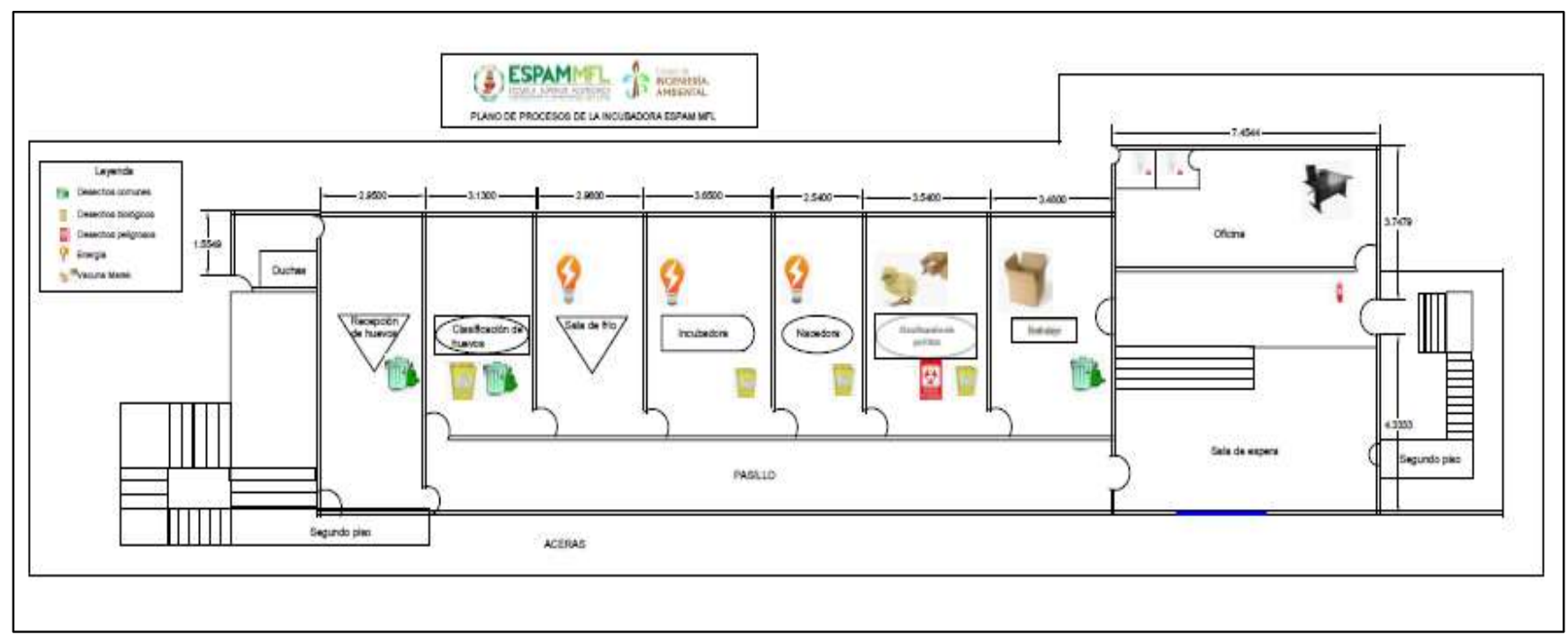

Figura 5. Plano de la Incubadora ESPAM

La evaluación de la actual distribución en planta de la incubadora de pollito BB ESPAM mostró que la superficie requerida para las instalaciones de la incubadora es de $186,66 \mathrm{~m}^{2}$ y su distribución ideal, de acuerdo al orden de importancia de los departamentos en función de la afinidad con todos los demás, sería:

Cuadro 4. Distribución ideal CORELAP

\begin{tabular}{clc}
\hline Orden & Actividad u operación & TCR \\
\hline 1 & Recepción de huevos & 30 \\
2 & Clasificación de huevo fértil & 28 \\
3 & Sala de frío & 26 \\
4 & Incubadora & 26 \\
5 & Nacedora & 24 \\
6 & Clasificación y vacunación de pollitos & 24 \\
7 & Embalaje & 24 \\
\hline
\end{tabular}

*TCR (total closeness rating)

La distribución gráfica según CORELAP sería (Figura 6):

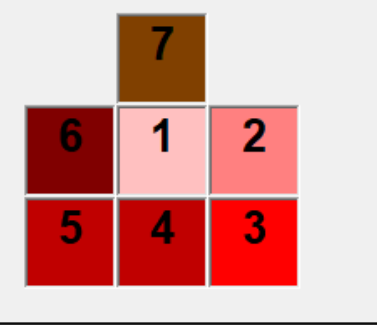

Figura 6. Layout adecuado CORELAP
Aunque el algoritmo CORELAP es útil para lograr un aprovechamiento máximo del espacio disponible, al aplicarlo a la planta incubadora de la ESPAM MFL mostró que el cuarto de recepción de huevos queda rodeado de los demás cuartos de procesos. Y según Vaca (2003) es conveniente que el área de descarga o cuarto de recepción de huevos esté cerca de la entrada de la planta para que los vehículos que transportan los huevos recorran el menor trecho posible dentro de las instalaciones ya que pueden ser considerados como principales focos de contaminación.

Se evidencia que la distribución por procesos de la incubadora es adecuada y aunque se podrían optimizar los niveles de producción, la distribución actual no es un limitante.

\section{CONCLUSIONES}

El análisis realizado en la incubadora de pollitos BB ESPAM MFL, muestra la pertinencia de la distribución por procesos que esta posee. La aplicación del algoritmo CORELAP demostró que, en el caso de la incubadora, una distribución por afinidad entre departamentos no es adecuada, pues no se ubica el cuarto de recepción de huevos cerca a la entrada de la planta. El enfoque presentado en este estudio es susceptible de usarse en la mejora de los niveles de producción de plantas incubadoras. 


\section{LITERATURA CITADA}

Akhila, P., y Deepthia, Y. 2019. A case study of Spinning Industry plant layout for effective production. India. Materials Today: Proceedings, 16:694-698.

Ali, S., Fahad, M., Atir, M., Zubair, M., y Shehzad, M. 2016. Productivity improvement of a manufacturing facility using systematic layout planning. Pakistan. Cogent Engineering, 3:1-13.

Autodesk Inventor. 2018. AutoCAD. Disponible en: https://latinoamerica.autodesk.com/products/autocad/ overview?term $=1$-YEAR

De Lira, J., López, A., Gutiérrez, C., y Vázquez, R. 2019. Optimal plant layout considerating the safety instrumented system design for hazardous equipment. México. Process Safety and Environmental Protection, 124: 97-120.

Delgado, M., Palacios, A., y González, J. 2013. Evaluación del desempeño ambiental de plantas incubadoras. Casos estudios: planta Incubadora de la carrera de pecuaria ESPAM MFL. II Evento Internacional "La Universidad en el Siglo XXI" Calceta-Ecuador:

Ejeh, J., Liu, S., y Papageorgiou, L. 2019. Optimal multifloor process plant layout with production sections. Chemical Engineering Research and Design, 137. DOI 488-501. doi: 10.1016/j.cherd.2018.07.018

González, L. 2002. Análisis de eficiencia y determinación de tiempos y movimientos de una planta incubadora. Honduras. Zamorano.

Guirardello, R., y Swaney, R. 2005. Optimization of process plant layout with pipe routing. MadisonEEUU. Computers and Chemical Engineering, 30: 99114.

Jung, B. y Lee, C. 2019. Plant and blast wall optimization with the consideration of operating conditions and potential explosions. Process Safety and Environmental Protection, 132: 285-293.
Latifi., S., Mohammadi, E., y Khakzad, N. 2017. Process Plant Layout Optimization with Uncertainty and Considering Risk. Iran. Computers and Chemical Engineering. 106:224-242

Mejía, H., Wilches, M., Galofre, M., y Montenegro, Y. 2011. Aplicación de metodologías de distribución de plantas para la configuración de un centro de distribución. Pereira-Colombia. Scientia et Technica, 16(49): 63-68.

Muther, R. 1968. Planificacion y proyeccion de la empresa industrial (método SLP). España. Editores tecnicos asociados, s. a.

Ning, X., Qi, J., Wu, C., y Wang, W. 2019. Reducing noise pollution by planning construction site layout via a multi-objective optimization model. China. Journal of Cleaner Production. 222:218-230.

Ojagh, Y., Khademi, A., Yusof, N., Renani, N., Helmi, S., y Hassan, S. 2005. Production Layout Optimization for Small and Medium Scale Food Industry. Malasia. Procedia CIRP. 26: 247-251.

Okpala, C., y Chukwumuanya, O. 2016. Plant layouts' analysis and design. Nigeria. International Journal of Advanced Engineering Technology. 7:201-206.

Patsiatzis, D., y Papageorgiou, L. 2002. Optimal multifloor process plant layout. Londres-Inglaterra. Computers and Chemical Engineering. 26: 575-583.

Vaca, L. 2003. Producción Avícola. Costa Rica: EUNED.

Yang, T., y Chih, H. 2007. Multiple-attribute decision making methods for plant layout design problem. Taiwan. Robotics and Computer-Integrated Manufacturing. 23:126-137. 